\title{
INFLUENCIA DEL CONTEXTO \\ EN PROBLEMAS DE MULTIPLICACIÓN \\ Y DIVISIÓN: ESTUDIO DE CASO \\ DE UN ALUMNO CON AUTISMO ${ }^{1}$
}

\section{Influence of the Context on Multiplication and Division Problems: A Case Study of a Student with Autism}

\author{
Irene Polo Blanco \\ Universidad de Cantabria. Departamento de Matemáticas, Estadística y Computación \\ gonzalelm@unican.es \\ María José GonzÁLez López \\ Universidad de Cantabria. Departamento de Matemáticas, Estadística y Computación \\ Alicia BRuno \\ Universidad de La Laguna. Departamento de Análisis Matemático
}

Recepción: 23 de enero de 2020

Aceptación definitiva: 14 de marzo de 2020

Resumen: Los estudiantes diagnosticados con Trastornos del Espectro Autista (TEA) suelen desarrollar un interés especial sobre áreas que no son habituales en cuanto a su intensidad o temática. Este trabajo profundiza en comprender la influencia que tienen las áreas de interés especial en los procesos de resolución de problemas aritméticos verbales de multiplicación y división, mediante un estudio de caso único con un estudiante de 11 años diagnosticado con TEA y discapacidad intelectual. Se utiliza un cuestionario formado por 15 problemas enunciados en tres tipos de contextos: de interés especial, familiar y no familiar. Siguiendo una metodología cualitativa, se clasifican las estrategias informales y el éxito en la obtención de la solución. Los resultados muestran que las áreas de interés especial han supuesto una mayor implicación del estudiante,

1 Este trabajo ha sido financiado por los proyectos de investigación con referencias EDU 201784276-R y PID2019-105677RB-I00 


\section{INFLUENCIA DEL CONTEXTO EN PROBLEMAS DE MULTIPLICACIÓN Y DIVISIÓN: ESTUDIO DE CASO DE UN ALUMNO CON AUTISMO \\ IRENE POLO BLANCO, MARÍA JOSÉ GONZÁLEZ LÓPEZ Y ALICIA BRUNO}

pero no han logrado una mejora efectiva respecto de los contextos familiares, ya que en ambos contextos el estudiante ha resuelto los problemas de multiplicación y de división-agrupamiento pero no ha logrado resolver los problemas de división-reparto. En el contexto no familiar no ha resuelto ningún problema. Estos hallazgos contribuyen a completar la literatura existente sobre la utilidad educativa de las áreas de interés especial en estudiantes diagnosticados con TEA.

Palabras clave: Trastorno del Espectro Autista; áreas de interés especial; contexto; problemas de multiplicación y división; estrategias informales de resolución de problemas.

Aвstract: Students diagnosed with Autism Spectrum Disorders (ASD) usually develop interest in areas that is unusual in its intensity or focus. This paper focuses on the influence of the special interest areas in the problem solving processes of multiplication and division word arithmetic problems, through a single case study with an 11-year-old student diagnosed with ASD and intellectual disability. A questionnaire consisting of 15 problems contextualized in three types of contexts -special interest context, a familiar context and an unfamiliar context- was applied. Following a qualitative method, informal strategies and success in obtaining the solution were registered. The results show that both, the special interest context and the familiar context, determined the success in obtaining the problem solution; the student did not solved any problem in the unfamiliar context; the special interest context led to a greater involvement of the student in the search of the solution, but it did not represent an effective improvement with respect to familiar contexts, since in both contexts the student solved the multiplication and division-grouping problems, but did not solve the partitive-division problems. These findings contribute to completing the existing literature on the educational potential of special interest areas in students diagnosed with ASD.

KEYwORDs: Autism Spectrum Disorder; special interest areas; context; multiplication and division problems; problem solving informal strategies.

\section{Introducción}

$\mathrm{D}$

ISTINTOS ESTUDIOS CONFIRMAN QUE EL CONOCIMIENTO de los estudiantes sobre el contexto que se presenta en el enunciado de un problema de matemáticas es un factor influyente a la hora de resolverlo (ver, por ejemplo, Almuna Salgado, 2017). Vicente y Orrantia (2007) llevaron a cabo una revisión de estudios en los que se analiza cómo la aplicación de conocimientos sobre el mundo real interviene en la resolución de problemas, concluyendo que, si bien la información situacional por sí misma no es útil para que los alumnos resuelvan con mayor acierto los problemas aritméticos verbales, cuando esta información permite hacer inferencias sobre la estructura matemática del problema, ayuda a los estudiantes a mejorar su rendimiento.

Los trabajos sobre resolución de problemas aritméticos verbales en estudiantes con Trastornos del Espectro Autista (TEA) han mostrado que estos estudiantes tienen dificultades para comprender el contexto al que hace referencia el enunciado (Bae, Chiang y Hickson, 2015), al tiempo que suelen desarrollar conocimiento profundo

Ediciones Universidad de Salamanca / CC BY-NC-ND

Siglo Cero, vol. 52 (1), 2021, enero-marzo, pp. 59-78 


\section{INFLUENCIA DEL CONTEXTO EN PROBLEMAS DE MULTIPLICACIÓN Y DIVISIÓN: ESTUDIO DE CASO DE UN ALUMNO CON AUTISMO \\ IRENE POLO BLANCO, MARÍA JOSÉ GONZÁLEZ LÓPEZ Y ALICIA BRUNO}

sobre algunos contextos particulares. Uno de los criterios que se tienen en cuenta para el diagnóstico de TEA es el interés especial que los sujetos desarrollan sobre algunas cuestiones, denominadas áreas de interés especial (Giarelli et al., 2010). Las áreas de interés especial se han utilizado como una herramienta eficaz en el desarrollo de habilidades sociales (Boyd, Conroy, Mancil, Nakao y Alter, 2007; Mancil y Pearl, 2008) y en el aprendizaje en ciertas áreas curriculares (Gunn y Delafield-Butt, 2016).

Este trabajo plantea un estudio de caso para determinar si las áreas de interés especial influyen en el proceso de resolución de problemas aritméticos verbales que lleva a cabo un estudiante de 11 años y 8 meses diagnosticado con TEA y discapacidad intelectual. Si bien la metodología de estudio de caso no permite establecer generalizaciones, es una de las pocas vías posibles para profundizar en la comprensión del aprendizaje en estudiantes con necesidades específicas de apoyo educativo debido a la gran variabilidad de características que presenta esta población. Además, el trabajo contribuye a completar un inventario de casos cada vez mayor, lo que proporciona una visión más global del tema analizado. Nos centramos en problemas aritméticos verbales de isomorfismo de medidas por ser los más habituales en el ámbito escolar cuando se aprende la estructura multiplicativa (Vicente, Manchado y Verschaffel, 2018). Nuestra contribución considera las operaciones de multiplicar y dividir, operaciones aritméticas que están poco analizadas en estudiantes diagnosticados con TEA. Adoptamos el proceso de modelización para problemas verbales de Verschaffel et al. (2000), según el cual los sujetos desarrollan un modelo de la situación a partir del enunciado verbal de un problema que sustenta sus estrategias de resolución e interactúa con el modelo matemático. El modelo de la situación representa los agentes, las acciones y las relaciones entre los acontecimientos que se describen en el enunciado del problema. En el caso de los problemas aritméticos verbales, este modelo explica el hecho de que, antes de haber recibido instrucción formal sobre las operaciones aritméticas, los estudiantes sean capaces de encontrar la solución numérica a un problema aritmético verbal utilizando estrategias informales basadas en las acciones que se describen en el problema. Dentro de las estrategias informales en los problemas aritméticos verbales de multiplicar y dividir se dan diferentes niveles de complejidad, dependiendo del método de conteo que utilicen los estudiantes y del uso que hagan de los objetos que se describen en el enunciado; el nivel más bajo es aquel en el que la técnica de cálculo se basa en el conteo de objetos concretos o dibujados y el nivel más alto es aquel en el que se emplean hechos numéricos (Mulligan, 1992; Kouba, 1989).

A través de las estrategias empleadas y del éxito en la obtención de la solución, analizamos el proceso de resolución de problemas de multiplicación y de división que lleva a cabo un estudiante diagnosticado con TEA y discapacidad intelectual cuando se le plantean problemas en tres tipos de contextos, que denominamos de interés especial, familiar y no familiar, diferenciados según los distintos grados de conocimiento que tiene el estudiante sobre la temática que se plantea en el enunciado. Los resultados obtenidos nos permiten dar respuesta a las cuestiones siguientes: ¿Se resuelve más fácilmente un problema aritmético verbal de multiplicar o dividir cuando el contexto del problema es un área de interés especial para el estudiante con TEA? ¿Qué diferencias hay en las estrategias de resolución según el tipo de contexto del problema? Las respuestas a estas cuestiones nos resultan útiles para ayudar a realizar diseños de instrucción que tomen en cuenta los tipos de contexto más adecuados para cada estudiante. 


\section{INFLUENCIA DEL CONTEXTO EN PROBLEMAS DE MULTIPLICACIÓN Y DIVISIÓN: ESTUDIO DE CASO DE UN ALUMNO CON AUTISMO IRENE POLO BLANCO, MARÍA JOSÉ GONZÁLEZ LÓPEZ Y ALICIA BRUNO}

\section{Referentes teóricos}

Los referentes teóricos de este trabajo se sustentan en los modelos cognitivos de resolución de problemas que contemplan la comprensión del enunciado del problema como parte del proceso de resolución, en el contexto de un problema como factor influyente en el proceso de resolución y en los tipos de estrategias de resolución de problemas aritméticos verbales de multiplicación y división.

\subsection{Modelos cognitivos de resolución de problemas}

Entre los modelos que, desde la psicología cognitiva, explican cómo los estudiantes resuelven problemas matemáticos contextualizados, destacan los que contemplan la comprensión de la situación descrita en el enunciado como parte del proceso de resolución (Van Dijk y Kintsch, 1983; Reusser, 1989; Stern y Lehrndorfer, 1992; Verschaffel et al., 2000). Estos modelos asumen que, a partir del enunciado de un problema, el sujeto desarrolla un modelo de la situación que representa los agentes, las acciones y las relaciones entre los acontecimientos que se describen en el enunciado del problema. El modelo de la situación juega un papel importante en el proceso de resolución del problema, interactúa con el modelo matemático y está asociado a la interpretación de las soluciones numéricas obtenidas. Antes de recibir instrucción formal sobre las operaciones aritméticas, los estudiantes pueden resolver una variedad de problemas mediante estrategias basadas en el modelo de la situación. Estas estrategias se ponen de manifiesto cuando, para encontrar la solución, los estudiantes hacen alguna simulación de la acción descrita en el problema junto con algún procedimiento de conteo (Carpenter et al., 1993; Mulligan y Mitchelmore, 1997; Brissiaud y Sander, 2010). Por ejemplo, los estudiantes encuentran la solución a un problema de dividir mediante una acción de reparto. Estas estrategias suelen denominarse informales y se consideran esenciales en el proceso de aprendizaje de las operaciones aritméticas. Incluso después de haber recibido instrucción formal sobre operaciones aritméticas, los estudiantes siguen empleando estrategias informales (Brissiaud y Sander, 2010). En estos casos, el modelo de la situación actúa como principal referente durante el proceso de resolución de un problema y las acciones que aparecen en el enunciado permiten a los estudiantes realizar actividades con sentido para obtener la solución del problema. Brissiaud y Sander (op. cit.) demuestran que, solo cuando el modelo de la situación no resulta eficiente para proporcionar la solución a un problema, los estudiantes desarrollan otro tipo de estrategias, denominadas estrategias aritméticas, asociadas al modelo matemático, que se caracterizan por el uso de propiedades matemáticas de las operaciones aritméticas (como la inversión o la propiedad conmutativa) y con la ejecución explícita de los algoritmos de dichas operaciones. Por ejemplo, ante un problema de dividir con números grandes, los estudiantes ya no podrán hacer un reparto, sino que tendrán que reconocer la operación aritmética que resuelve el problema y efectuar el algoritmo de la división. 


\section{INFLUENCIA DEL CONTEXTO EN PROBLEMAS DE MULTIPLICACIÓN Y DIVISIÓN: ESTUDIO DE CASO DE UN ALUMNO CON AUTISMO \\ IRENE POLO BLANCO, MARÍA JOSÉ GONZÁLEZ LÓPEZ Y ALICIA BRUNO}

\subsection{El contexto del problema y las áreas de interés especial en TEA}

La expresión contexto del problema ha recibido significados muy diversos (Brown, 2019). En este estudio utilizamos problemas aritméticos verbales de una etapa en los que se proporcionan dos datos numéricos en una situación real o simulada sobre alguna temática, y se realiza una pregunta que puede responderse a partir de alguna acción o de alguna operación aritmética sobre dichos datos. Considerando estos problemas y el objetivo de este estudio, denominaremos contexto del problema a la temática de la situación que aparece en el enunciado.

Distinguiremos distintos tipos de contextos atendiendo al grado de proximidad de la temática a las experiencias del estudiante (Almuna Salgado, 2017). Utilizando este criterio, Huang (2004) define un problema en contexto familiar para un estudiante como aquel en el que los elementos y entornos reales presentados en el problema se extraen de las experiencias diarias y de las actividades escolares del estudiante, y un problema en contexto no familiar como aquel en el que los elementos presentados en el problema son desconocidos para el estudiante. En general, se ha observado que los contextos familiares facilitan el desarrollo de modelos de la situación eficaces (Stern y Lehrndorfer, 1992). Por ejemplo, si se pide a un estudiante "calcular la gasolina que consume un coche, sabiendo que se le pone gasolina 4 veces al mes, echando 15 litros cada vez", resultará más complejo que si se le propone "calcular los minutos que tardará en leer 4 cuentos, sabiendo que tarda 15 minutos en cada cuento". Los contextos no familiares pueden conllevar cambios en la demanda cognitiva de los problemas, aunque estos tengan la misma estructura semántica. Así, Fernández Bravo (2007) pone de manifiesto que el concepto de "veces", junto con la expresión "cada uno", son apoyos para realizar la multiplicación mediante estrategias informales. Pero cuando el contexto es desconocido para el estudiante, comprender una situación en términos del número de veces no resulta sencillo. La complejidad debida al conocimiento situacional engloba multitud de factores. Seguidamente nos centraremos en los estudiantes diagnosticados con TEA para profundizar en esta idea.

Los estudiantes diagnosticados con TEA suelen presentar dificultades para comprender situaciones en las que se describen temáticas desconocidas (Bae et al., 2015). Merecen atención particular las situaciones en las que aparece, implícita o explícitamente, alguna norma o habilidad social. Por ejemplo, en las situaciones de manejo de dinero se requiere que el estudiante haya desarrollado un conocimiento con un componente social, como es la asignación de valor a los objetos. Por otro lado, en sentido positivo, los estudiantes con TEA pueden llegar a desarrollar conocimiento profundo sobre ciertas temáticas. Según el DSM-5 (APA, 2013), uno de los criterios que se utilizan para el diagnóstico del TEA es la presencia en el sujeto de áreas de interés especial, también llamado interés restringido. Este criterio se manifiesta cuando el sujeto tiene un interés acusado en cuestiones que no son habituales, ni en su temática ni en su intensidad (Giarelli et al., 2010).

En los últimos años se está tratando de utilizar las áreas de interés especial como una herramienta para mejorar distintos aspectos del aprendizaje de los estudiantes diagnosticados con TEA (Boyd et al., 2007; Mancil y Pearl, 2008). Gunn y Delafield- 


\section{INFLUENCIA DEL CONTEXTO EN PROBLEMAS DE MULTIPLICACIÓN Y DIVISIÓN: ESTUDIO DE CASO DE UN ALUMNO CON AUTISMO \\ IRENE POLO BLANCO, MARÍA JOSÉ GONZÁLEZ LÓPEZ Y ALICIA BRUNO}

Butt (2016) han examinado los estudios publicados sobre este tema entre 1990 y 2014, concluyendo que, cuando se utilizan adecuadamente las áreas de interés especial, se producen mejoras en el rendimiento académico y/o las habilidades sociales en la mayoría de los casos. Mancil y Pearl (2008) muestran un caso en el que un estudiante diagnosticado con TEA mejora su rendimiento en problemas de sumar cuando se contextualiza la operación en una de sus áreas de interés especial (trenes). Nuestro propósito es seguir profundizando en esta línea de investigación, focalizándola en problemas de multiplicar y dividir.

Asumiendo el planteamiento de Huang (2004), en este trabajo distinguimos el contexto familiar y no familiar, y añadimos otro tipo de contexto vinculado a las áreas de interés especial para los estudiantes diagnosticados con TEA. Así, se consideran en este trabajo los problemas siguientes:

- Problema en contexto de interés especial: la temática del problema no tiene por qué estar relacionada con las experiencias diarias y las actividades escolares del estudiante, pero constituye un ámbito sobre el que el sujeto se ha interesado de manera particular y ha desarrollado un conocimiento intenso e inusual.

- Problema en contexto familiar: la temática es conocida y cercana para el estudiante por estar relacionada con experiencias diarias y actividades escolares, pero no es un área de interés especial.

- Problema en contexto no familiar: la temática no es de interés especial ni familiar para el estudiante.

\subsection{Problemas de isomorfismo de medidas}

Puesto que nuestro propósito es determinar qué diferencias se perciben en el proceso de resolución de problemas según los tres tipos de contextos planteados, hemos evitado la influencia de otros factores fijando un tipo de problemas aritméticos verbales de una etapa que responden a la misma estructura semántica: los problemas de isomorfismo de medidas (Vergnaud, 1997). En estos problemas aparecen dos cantidades que se relacionan mediante una regla de proporcionalidad directa, por ejemplo, "Hay 5 estantes de libros en la habitación de Juan. Juan puso 8 libros en cada estante. Juan tiene 40 libros en su habitación”. Cuando la incógnita es el producto (40 libros) tenemos un problema de multiplicación, cuando la incógnita es el multiplicando (8 libros/estante) tenemos un problema de división-reparto y cuando la incógnita es el multiplicador (5 estantes) tenemos un problema de división-agrupamiento.

\subsection{Estrategias informales en problemas de isomorfismo de medidas}

Mulligan (1992) y Kouba (1989) han proporcionado clasificaciones detalladas de las estrategias informales que emplean los estudiantes al resolver problemas verbales de multiplicar y dividir basándose en dos criterios: el grado de abstracción del procedimiento empleado al calcular y el modo en que los objetos propios del enunciado de 


\section{INFLUENCIA DEL CONTEXTO EN PROBLEMAS DE MULTIPLICACIÓN Y DIVISIÓN: ESTUDIO DE CASO DE UN ALUMNO CON AUTISMO \\ IRENE POLO BLANCO, MARÍA JOSÉ GONZÁLEZ LÓPEZ Y ALICIA BRUNO}

los problemas se usan durante el proceso de resolución. Combinando estos criterios se describen los tipos de estrategias siguientes, que se presentan ordenadas desde un nivel bajo, en el que la técnica de cálculo se basa en el conteo de objetos concretos o dibujados, hasta un nivel alto, en el que la solución se obtiene mediante el uso de hechos numéricos sin el apoyo de objetos o dibujos:

- Modelización directa con conteo: el estudiante hace uso de objetos concretos o dibujos para representar la acción descrita en el problema. Obtiene la solución utilizando distintas técnicas de conteo. Por ejemplo, para calcular cuántas bombillas hay en 4 farolas sabiendo que cada farola tiene tres bombillas, el estudiante hace el dibujo de las 4 farolas, en cada farola dibuja 3 bombillas y finalmente hace un conteo del total de bombillas dibujadas (Ivars y Fernández, 2016).

- Conteo sin modelización: se realizan las mismas acciones que en el nivel anterior, pero sin utilización de objetos o dibujos, sino que el estudiante encuentra la respuesta mediante una acción mental. Estas estrategias incluyen la posibilidad de hacer conteos rítmicos ascendentes o descendentes. Por ejemplo, en el problema anterior, el estudiante encuentra la respuesta escribiendo: 3-6-9-12.

- Hechos numéricos conocidos o derivados: se utilizan hechos numéricos para alcanzar el resultado de la multiplicación o la división. Por ejemplo, en el problema anterior, el estudiante argumenta que 3 x 3 son 9, y 3 más son 12 .

Además, tomaremos la siguiente subclasificación de estrategias de modelización directa con conteo (Caballero, 2005 y Rodríguez et al., 2008), por ser las más habituales para el nivel del estudiante objeto de este estudio. De nuevo, presentamos estas estrategias ordenadas según la complejidad de la técnica de cálculo implicada.

En problemas de multiplicación:

- Multiplicación por adición repetida

- Con representación uno a uno (M-N1). Formar tantos grupos como indica el multiplicador y colocar, uno a uno en cada grupo, los elementos correspondientes al multiplicando. Finalizar contando todos los elementos.

- Con representación por múltiplos (M-N2). Formar tantos grupos como indica el multiplicador y añadir a cada grupo los elementos que indica el multiplicando. No se pasa al siguiente grupo hasta que no se ha completado el anterior. Finalizar contando todos los elementos.

- Con representación uno a uno sin recuento final (M-N3). Representar el multiplicando y añadir los elementos del multiplicador al tiempo que se van contando (no se necesita volver a contar al final).

- Con representación de un único factor sin recuento final (M-N4). Representar solo el multiplicando y añadir mentalmente los elementos del multiplicador.

En problemas de división:

- División reparto. Representar el dividendo y repartir los elementos, formando los conjuntos que indica el divisor.

- Por ensayo-error (DR-N1). Repartir al azar y redistribuir los elementos repartidos hasta igualar los conjuntos.

- De uno en uno (DR-N2). Repartir asignando cada vez una unidad. 


\section{INFLUENCIA DEL CONTEXTO EN PROBLEMAS DE MULTIPLICACIÓN Y DIVISIÓN: ESTUDIO DE CASO DE UN ALUMNO CON AUTISMO IRENE POLO BLANCO, MARÍA JOSÉ GONZÁLEZ LÓPEZ Y ALICIA BRUNO}

- Por múltiplos (DR-N3). Repartir asignando una cantidad fija mayor que uno y repetir el proceso si no se han agotado los elementos del dividendo.

- División adición repetida. Se forma un primer conjunto con la cantidad correspondiente al divisor y se añaden conjuntos equivalentes hasta alcanzar el dividendo. Estas estrategias se secuencian, a su vez, en los siguientes niveles:

- De representar y contar (DA-N1). Representar grupos del tamaño del divisor y a continuación hacer un recuento para comprobar si se ha alcanzado el dividendo. Finalmente, contar el número de grupos formados.

- De representar con doble conteo (DA-N2). Hacer grupos del tamaño del divisor hasta alcanzar el dividendo llevando la cuenta de los grupos que se forman.

- De representar un único término y proseguir mentalmente (DA-N3). Comenzar representando el dividendo o el divisor y continuar el reparto mentalmente.

\section{Metodología}

Se utiliza una metodología cualitativa de estudio de caso único. Tiene un propósito descriptivo orientado a analizar en profundidad las características particulares de la resolución de problemas de matemáticas planteados en distintos contextos por alumnado diagnosticado con TEA.

\subsection{Participante}

El sujeto de la investigación es un varón de 11 años y 8 meses. Fue diagnosticado con TEA a la edad de 6 años a partir de evaluaciones clínicas y según los criterios de diagnóstico del DSM-IV (López-Ibor Aliño y Valdés Miyar, 2002). Según la Escala de Evaluación de la Actividad Global presentó un nivel de afectación entre 41 y 50, que corresponde a sintomatología grave con alteración grave de la actividad social, laboral o escolar. Presenta un repertorio amplio de conductas estereotipadas, muestra tendencia a conductas repetitivas y tiene interés especial por ciertas áreas, dos de las cuales -aviones y molinos de viento- serán utilizadas en los problemas que se le van a plantear. Estuvo escolarizado en un colegio ordinario hasta $4 .^{\circ}$ de Primaria con una adaptación curricular individual significativa en las áreas de lengua y matemáticas. Desde los 10 años está escolarizado en un centro de educación especial. En matemáticas sigue un currículo adaptado al que dedica 4 horas semanales. Según el último informe del equipo de orientación del centro, dentro de la sociabilidad y los aspectos emocionales se muestra cercano con sus iguales, aunque con escasa integración en el juego o en la relación. Tiene buena reacción ante las rutinas establecidas y le tranquiliza prever con antelación lo que va a ocurrir. En la estructuración de su personalidad hay un estancamiento en el desarrollo emocional y cognitivo, con afectación del proceso simbólico. Se comunica por medio de frases cortas gramaticalmente correctas. Su 


\section{INFLUENCIA DEL CONTEXTO EN PROBLEMAS DE MULTIPLICACIÓN Y DIVISIÓN: ESTUDIO DE CASO DE UN ALUMNO CON AUTISMO IRENE POLO BLANCO, MARÍA JOSÉ GONZÁLEZ LÓPEZ Y ALICIA BRUNO}

capacidad lectora es equiparable a un nivel de tercero de Primaria (8-9 años). Ello le permite leer y entender los enunciados de los problemas, aunque presenta dificultades de comprensión de determinadas palabras.

Presenta discapacidad intelectual, con CI de 54 según la escala WISC-V de inteligencia para niños, desglosado según se indica en la Tabla 1. Familiares del estudiante y profesores especialistas que han trabajado con él le describen como un pensador visual (Grandin, 2008), es decir, su razonamiento se apoya en imágenes fotográficas. Al comienzo del estudio se le aplicó el Test de Competencia Matemática Básica TEMA-3, dirigido a evaluar la competencia matemática evaluando aspectos matemáticos informales (numeración, comparación...) y formales (cálculo y hechos numéricos de suma y resta, principalmente). El participante obtuvo una puntuación directa de 56 (Tabla 1), equivalente a una edad matemática de 7 años y 8 meses. Tiene conocimientos de la serie numérica hasta el 100 y realiza el algoritmo de la suma y la resta sin llevadas de manera autónoma en casos sencillos. Ha trabajado la resolución de problemas de suma y resta de manera escrita (con dibujos y con algoritmos). Se ha iniciado en el aprendizaje de algunas multiplicaciones sencillas con números pequeños $(2 \times 3,3 \times 4$, etc.), para lo cual ha desarrollado el significado de suma reiterada mediante esquemas dibujados y conteo, pero no ha memorizado las tablas de multiplicar ni ha recibido instrucción formal sobre los algoritmos de multiplicar y dividir. Se encuentra, por tanto, en un momento adecuado para utilizar distintos tipos de estrategias informales en problemas multiplicativos de multiplicar y dividir, lo que nos permitirá identificar si la familiaridad con el contexto y las temáticas de interés especial influyen en el tipo de estrategia informal que elige.

\section{TABLA 1. Características del sujeto. CI Cociente intelectual, WISC-V Escala de inteligencia de Wechsler para niños-V, TEMA-3 Test de Competencia Matemática Básica}

\section{CI (WISC-V)}

\begin{tabular}{|l|l|} 
Escala completa & 54 \\
\hline Comprensión verbal & 55 \\
\hline Viso-espacial & 67 \\
\hline Razonamiento fluido & 64 \\
\hline $\begin{array}{l}\text { Memoria de } \\
\text { trabajo }\end{array}$ & 79 \\
\hline Velocidad de procesamiento & 45 \\
\hline
\end{tabular}




\begin{tabular}{|l|l|l|}
\hline Nivel de competencia matemática (TEMA-3) \\
\hline \multirow{4}{*}{ Pensamiento informal } & Puntación directa & 56 \\
\cline { 2 - 3 } & Edad matemática & $7: 8$ \\
\cline { 2 - 3 } & Numeración & 100 \\
\cline { 2 - 3 } & Comparación & 50 \\
\cline { 2 - 3 } & Cálculo & 88 \\
\cline { 2 - 3 } & Conceptos & 75 \\
\hline \multirow{4}{*}{ Pensamiento formal } & Convencionalismos & 100 \\
\cline { 2 - 3 } & Hechos numéricos & 89 \\
\cline { 2 - 3 } & Cálculo & 11 \\
\cline { 2 - 3 } & Conceptos & 20 \\
\hline
\end{tabular}

\subsection{Instrumento de recogida de datos}

Se diseñó un cuestionario formado por 15 problemas de isomorfismo de medidas de multiplicación y división de una etapa. Se emplearon números cuyo producto es máximo 40. El cuestionario se construyó utilizando las estructuras de enunciados y de cantidades de los problemas de Carpenter et al. (1993), adaptándolos a los tres tipos de contextos analizados en esta investigación. Más adelante se muestran varios de los problemas empleados.

Para saber los contextos que interesan a los estudiantes diagnosticados con TEA es necesaria una colaboración de los profesionales educativos y los padres (Bae et al., 2015). Por ello, se pidió a los padres del participante que detallaran temas que eran de interés especial para su hijo y los temas para los que, claramente, no mostrase interés en su vida cotidiana ni escolar. A partir de esa información se seleccionaron dos contextos asociados a áreas de interés especial -aviones y molinos- y un contexto no familiar-dinero-. También se les preguntó si determinados temas eran conocidos para su hijo, sin situarse en los extremos anteriores, lo que ayudó a seleccionar dos contextos de tipo familiar -perros y mesas-.

Se aplicaron 5 problemas de multiplicar, 5 de división-agrupamiento y 5 de división-reparto. La Tabla 2 muestra las denominaciones que utilizamos para ellos. 
INFLUENCIA DEL CONTEXTO EN PROBLEMAS DE MULTIPLICACIÓN Y DIVISIÓN: ESTUDIO DE CASO DE UN ALUMNO CON AUTISMO

IRENE POLO BLANCO, MARÍA JOSÉ GONZÁLEZ LÓPEZ Y ALICIA BRUNO

\begin{tabular}{|l|c|c|c|c|c|}
\hline \multicolumn{5}{|c|}{ TabLa 2. Clasificación de problemas según operación y contexto } \\
\hline & \multicolumn{5}{c|}{ Contextos } \\
\cline { 2 - 6 } & \multicolumn{2}{|c|}{ Interés especial } & \multicolumn{2}{c|}{ Familiar } & No familiar \\
\cline { 2 - 6 } & Molinos & Aviones & Perros & Mesas & Dinero \\
\hline Multiplicación & P1A & P2A & P3A & P4A & P5A \\
\hline División-agrup. & P1B & P2B & P3B & P4B & P5B \\
\hline División-reparto & P1C & P2C & P3C & P4C & P5C \\
\hline
\end{tabular}

Los dos profesores de apoyo escolar habituales y los padres del participante confirmaron que los contextos eran adecuados y la redacción de los enunciados comprensibles para el estudiante.

\subsection{Procedimiento de recogida de datos}

El estudiante resolvió el cuestionario en 5 sesiones de 1 hora, distribuidas a lo largo de dos meses, en una sala en la que se evitaron elementos de distracción. Se buscaron momentos en los que el estudiante estaba participativo y descansado. Cada sesión se dedicó a un contexto, comenzando por los temas de interés especial, siguiendo por los temas familiares y terminando por el tema no familiar. Así, en la sesión 1, el estudiante resolvió los problemas P1A, P1B y P1C sobre la temática de los molinos; en la sesión 2 resolvió los problemas P2A, P2B y P2C sobre los aviones; la sesión 3, con los problemas P3A, P3B y P3C, trató el tema de los perros; la sesión 4, con los problemas P4A, P4B y P4C, trató las mesas y los niños; y, por último, la sesión 5 trató el tema del dinero con los problemas P5A, P5B y P5C. Este orden nos pareció el más adecuado, debido a que comenzaba con temáticas motivadoras que darían pie al estudiante a buscar soluciones a los problemas. Cabía la posibilidad de que se produjese algún efecto de aprendizaje a lo largo de las sesiones, pero este efecto, precisamente, apoyaría la idea de que las áreas de interés especial podrían haber supuesto una ayuda para el progreso del estudiante. Veremos más adelante, cuando presentemos los resultados finalmente obtenidos, que no se observó dicho efecto de aprendizaje.

Las sesiones se grabaron en vídeo con el fin de observar los movimientos del estudiante y las comunicaciones verbales con la profesora. Esto complementó la información escrita y facilitó la identificación de las estrategias.

\subsection{Análisis de datos y confiabilidad entre evaluadores}

Se codificaron las respuestas del estudiante por dos investigadores de forma independiente. Registraron tipo de estrategia y éxito en la solución. La confiabilidad 
INFLUENCIA DEL CONTEXTO EN PROBLEMAS DE MULTIPLICACIÓN Y DIVISIÓN: ESTUDIO DE CASO DE UN ALUMNO CON AUTISMO

IRENE POLO BLANCO, MARÍA JOSÉ GONZÁLEZ LÓPEZ Y ALICIA BRUNO

(número de acuerdos entre número de acuerdos más desacuerdos) para la codificación de las estrategias fue del $93 \%$ con Kappa de Cohen de 0,91. Los desacuerdos fueron resueltos mediante observación conjunta de los vídeos y registros escritos.

\section{Resultados}

En la Tabla 3 se resumen los resultados del estudio agrupados por tipo de contexto y operación.

\begin{tabular}{|c|c|c|c|c|c|}
\hline Contexto & Operación & Prob. & \multicolumn{2}{|l|}{ Estrategia } & \multirow{2}{*}{\begin{tabular}{|l|} 
Éxito \\
E \\
\end{tabular}} \\
\hline \multirow{6}{*}{$\begin{array}{l}\text { Interés } \\
\text { especial }\end{array}$} & \multirow{2}{*}{ Multiplicar } & P1A & $\begin{array}{l}\text { Modelización directa } \\
\text { con conteo }\end{array}$ & M-N2 & \\
\hline & & $\mathrm{P} 2 \mathrm{~A}$ & $\begin{array}{l}\text { Modelización directa } \\
\text { con conteo }\end{array}$ & M- N1 & E \\
\hline & \multirow{2}{*}{ Dividir-agrup. } & P1B & $\begin{array}{l}\text { Modelización directa } \\
\text { con conteo }\end{array}$ & DA-N1 & E \\
\hline & & P2B & $\begin{array}{l}\text { Modelización directa } \\
\text { con conteo }\end{array}$ & DA-N1 & E \\
\hline & \multirow{2}{*}{ Dividir-reparto } & P1C & $\begin{array}{l}\text { Modelización directa } \\
\text { con conteo }\end{array}$ & DA-N1 & $\mathrm{F}$ \\
\hline & & $\mathrm{P} 2 \mathrm{C}$ & $\begin{array}{l}\text { Modelización directa } \\
\text { con conteo }\end{array}$ & - & $\mathrm{F}$ \\
\hline \multirow{6}{*}{ Familiar } & \multirow[b]{2}{*}{ Multiplicar } & $\mathrm{P} 3 \mathrm{~A}$ & \multicolumn{2}{|c|}{ Conteo sin modelización } & E \\
\hline & & P4A & $\begin{array}{l}\text { Modelización directa } \\
\text { con conteo }\end{array}$ & M-N2 & $\mathrm{E}$ \\
\hline & Dividir-agrup. & P3B & $\begin{array}{l}\text { Modelización directa } \\
\text { con conteo }\end{array}$ & DA-N3 & E \\
\hline & & $\mathrm{P} 4 \mathrm{~B}$ & \multicolumn{2}{|c|}{ Conteo sin modelización } & $E$ \\
\hline & & P3C & \multicolumn{2}{|c|}{-} & $\mathrm{B}$ \\
\hline & Dividir-reparto & $\mathrm{P} 4 \mathrm{C}$ & \multicolumn{2}{|l|}{-} & $\mathrm{B}$ \\
\hline \multirow{3}{*}{$\begin{array}{l}\text { No } \\
\text { familiar }\end{array}$} & Multiplicar & P5A & \multicolumn{2}{|l|}{-} & $\mathrm{B}$ \\
\hline & Dividir-agrup. & P5B & \multicolumn{2}{|l|}{-} & $\mathrm{B}$ \\
\hline & Dividir-reparto & P5C & \multicolumn{2}{|l|}{-} & $\mathrm{B}$ \\
\hline
\end{tabular}

M-N1: multiplicación por adición repetida representación uno a uno. M-N2: multiplicación por adición repetida representación por múltiplos. DA-N1: división por adición repetida, representar y contar. DA-N3: división por adición repetida, representar un término y proseguir mentalmente. E: Éxito. F: Fracaso. B: Blanco. 


\section{INFLUENCIA DEL CONTEXTO EN PROBLEMAS DE MULTIPLICACIÓN Y DIVISIÓN: ESTUDIO DE CASO DE UN ALUMNO CON AUTISMO \\ IRENE POLO BLANCO, MARÍA JOSÉ GONZÁLEZ LÓPEZ Y ALICIA BRUNO}

El estudiante tuvo éxito en los problemas de contexto de interés especial y familiar en los problemas de multiplicación y de división-agrupamiento. No resolvió correctamente los problemas de división-reparto en ninguno de los tres contextos. En relación con las estrategias que empleó, y comparando los contextos familiar y de interés especial, los contextos de interés especial no supusieron una ayuda para emplear estrategias más eficientes, sino que en ellos empleó estrategias de niveles más bajos que en los contextos de interés familiar. Seguidamente detallamos estos resultados según cuál sea la incógnita del problema (producto, multiplicando o multiplicador).

\subsection{Resultados en problemas de multiplicación}

El alumno tuvo éxito en 4 de los 5 problemas de multiplicación, predominando las estrategias de modelización directa con conteo (P1A, P2A y P4A). En los problemas P1A y P4A (Figura 1) sigue la estrategia de adición repetida con representación por múltiplos (M-N2). Dibuja los elementos del multiplicando (bases de los molinos en P1A y mesas en P4A) y seguidamente dibuja el multiplicador en cada uno de los elementos (tres aspas por base y cuatro niños por mesa). Al finalizar el dibujo, realiza un recuento final de todos los objetos y escribe la respuesta.

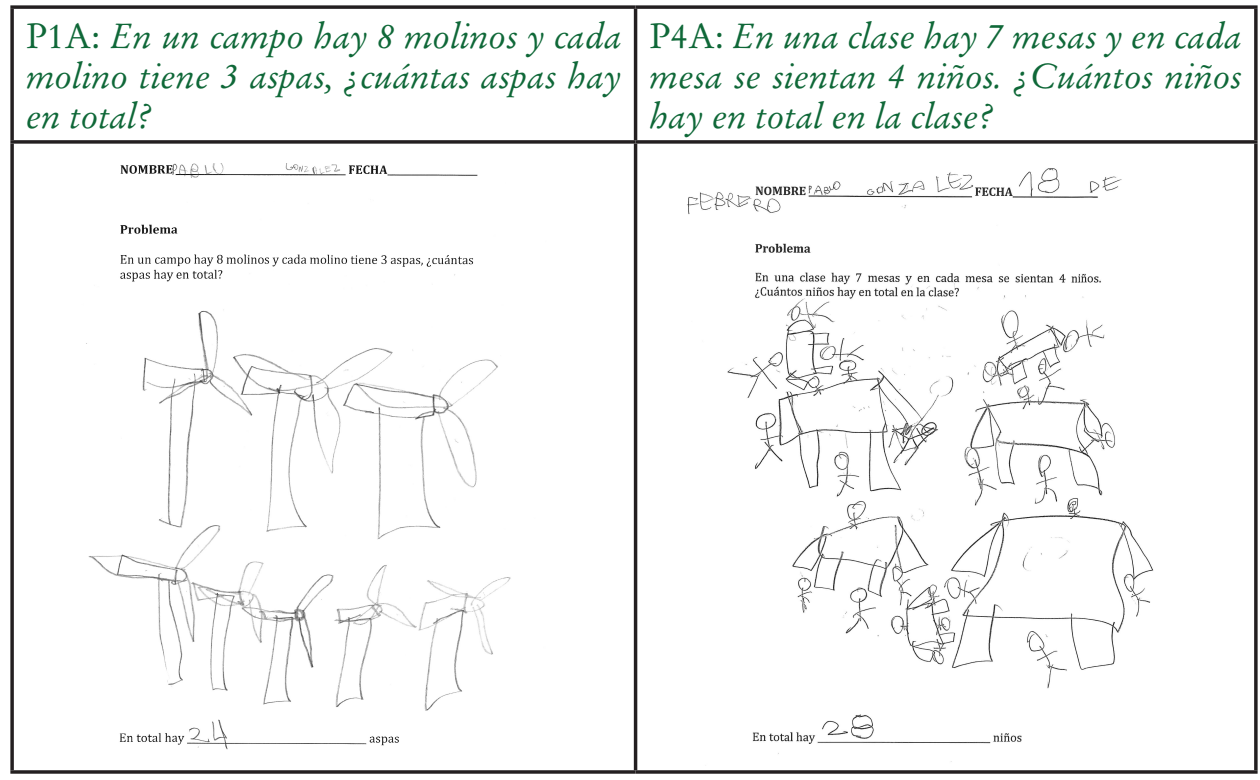

Figura 1. Resolución de los problemas P1A y P4A. 


\section{INFLUENCIA DEL CONTEXTO EN PROBLEMAS DE MULTIPLICACIÓN Y DIVISIÓN: ESTUDIO DE CASO DE UN ALUMNO CON AUTISMO IRENE POLO BLANCO, MARÍA JOSÉ GONZÁLEZ LÓPEZ Y ALICIA BRUNO}

En el problema P2A (Figura 2) el estudiante comienza dibujando los 8 aviones, después coloca un motor a cada avión y sigue dibujando motores de forma aleatoria hasta que cada avión tiene 4 motores. Después realiza un conteo rítmico $(1,2,3,4$, pausa, $5,6,7,8$, pausa...) hasta llegar a 32. Dice la respuesta correcta, escribe los números sobre cada motor y termina escribiendo la respuesta.

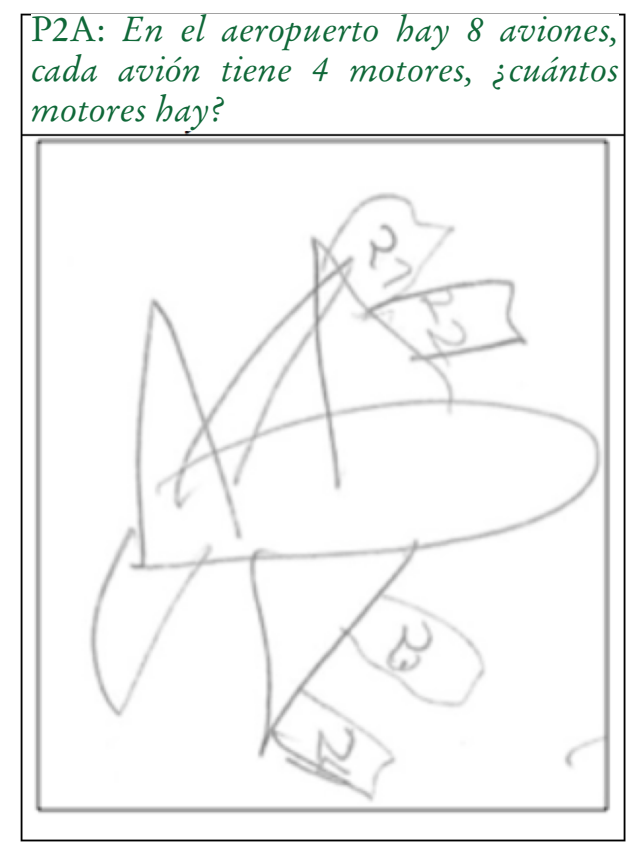

FIgURA 2. Ejemplo parcial de modelización directa con representación uno a uno y recuento final (M-N1) en problema P2A.

El estudiante resuelve el problema P3A (En una perrera hay 7 perros y cada perro ha dado 3 ladridos ¿cuántos ladridos han dado en total?) sin realizar ningún registro escrito hasta el final. Comienza realizando un conteo rítmico marcando tres movimientos en los dedos $(1,2,3-4,5,6-7,8,9 \ldots)$. Al finalizar con el séptimo dedo escribe la respuesta correcta, 21. Así evidencia que es capaz de realizar estrategias de multiplicación más evolucionadas sin apoyo en dibujos, aunque solo lo ha hecho en un contexto que no forma parte de sus áreas de interés especial. En el área de interés especial (molinos y aviones) el estudiante se recrea dibujando detalles y emplea estrategias más rudimentarias en la multiplicación.

El estudiante no resuelve el problema P5A de contexto no familiar (He comprado 6 bombillas. Cada bombilla me ha costado 3 euros. ¿Cuántos euros me he gastado?). Tras leer el enunciado, se muestra confuso y comienza a decir números al azar, dejando finalmente la respuesta en blanco. 


\section{INFLUENCIA DEL CONTEXTO EN PROBLEMAS DE MULTIPLICACIÓN Y DIVISIÓN: ESTUDIO DE CASO DE UN ALUMNO CON AUTISMO IRENE POLO BLANCO, MARÍA JOSÉ GONZÁLEZ LÓPEZ Y ALICIA BRUNO}

\subsection{Resultados en problemas de división-agrupamiento}

El alumno resolvió con éxito 4 de los 5 problemas de división por agrupamiento y dejó sin resolver el problema del contexto no familiar. Resolvió los 2 problemas planteados en áreas de interés especial utilizando una estrategia de modelización directa de primer nivel (DA-N1), mientras que en contexto familiar usó estrategias más avanzadas (DA-N3 y conteo sin modelización). Por ejemplo, en P1B (Figura 3, izda.) dibujó primero grupos de tres aspas de molino. Cuando llevaba dibujados 6 grupos, se detuvo y contó las aspas dibujadas (18 aspas). Continuó contando de nuevo todas las aspas cada vez que añadía un grupo de 3 aspas. Al terminar, dibujó las bases de los molinos. Cuando los 9 molinos estaban dibujados, los contó y escribió la respuesta correcta, 9 molinos. Utilizó, por tanto, la estrategia DA-N1.

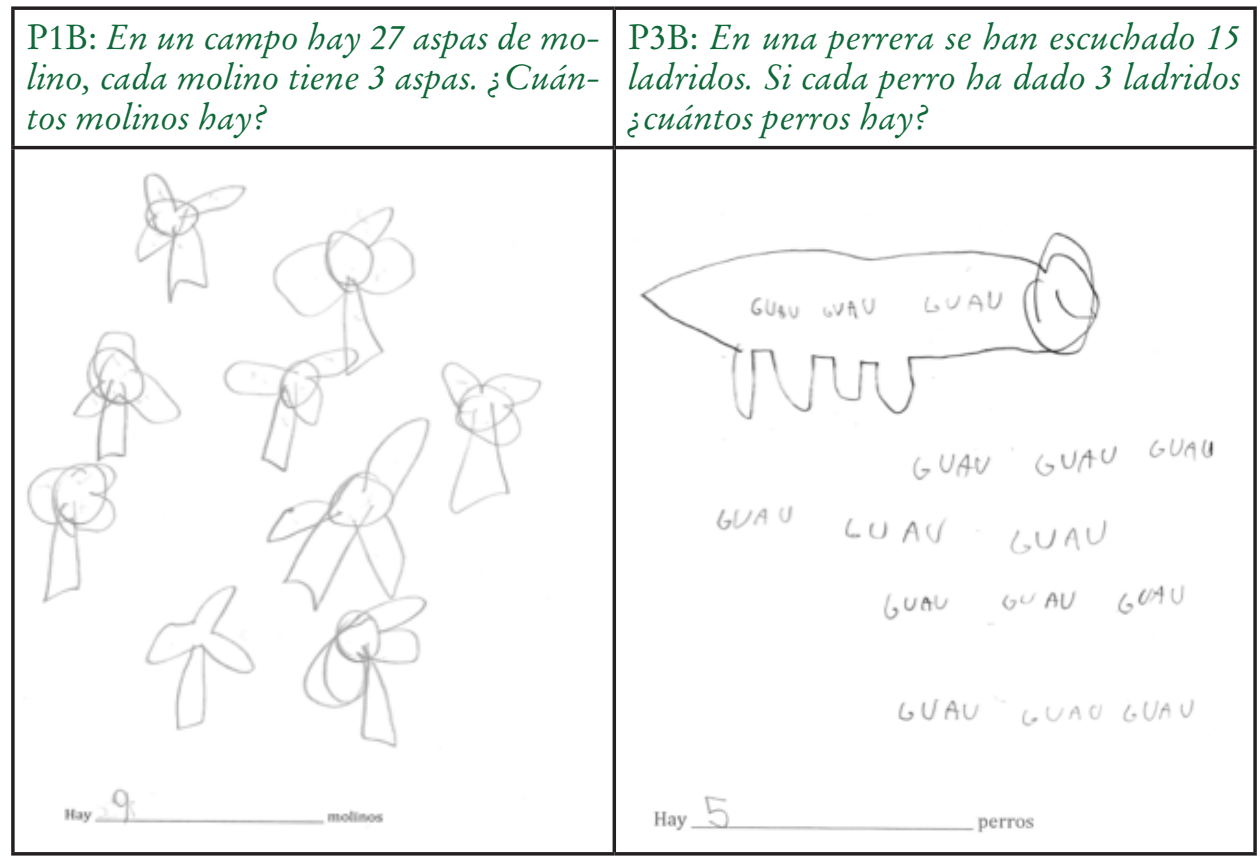

Figura 3. Resolución de los problemas P1B y P3B.

En P3B, de contexto familiar (Figura 3, dcha.), manifestó una estrategia más avanzada. Comenzó escribiendo los 15 ladridos de tres en tres (representado cada uno por la onomatopeya “guau”) en diferentes filas. A continuación, dibujó uno de los perros englobando tres ladridos y escribió la respuesta correcta (5 perros). Interpretamos que hizo un recuento mental de los grupos de ladridos. Se manifiesta, en este caso, una estrategia de adición repetida de nivel 3, en la que representa un único múltiplo y 


\section{INFLUENCIA DEL CONTEXTO EN PROBLEMAS DE MULTIPLICACIÓN Y DIVISIÓN: ESTUDIO DE CASO DE UN ALUMNO CON AUTISMO \\ IRENE POLO BLANCO, MARÍA JOSÉ GONZÁLEZ LÓPEZ Y ALICIA BRUNO}

prosigue mentalmente (DA-N3). Este uso de una estrategia más evolucionada se confirma en el otro problema de contexto familiar, P4B (En una clase hay 16 niños y en cada mesa se sientan 4 niños. ¿Cuántas mesas hay en la clase?). El estudiante lo resolvió mediante una estrategia de conteo sin modelización, que se inició con un conteo rítmico con los dedos en grupos de 4 y continuó con el mismo recuento mentalmente. Al igual que en los problemas de multiplicación, en el área de interés especial el estudiante se ha recreado dibujando detalles y ha empleado estrategias más primarias.

El estudiante no resolvió el problema de contexto no familiar P5B (He comprado bombillas y me he gastado 24 euros. Cada bombilla cuesta 4 euros. ¿Cuántas bombillas he comprado?). Tras leerlo mostró confusión y dejó la respuesta en blanco.

\subsection{Resultados en problemas de división-reparto}

Ninguno de los 5 problemas de división de reparto fue resuelto correctamente por el estudiante, pero se apreció una diferencia según el tipo de contexto: en los problemas de interés especial hizo dibujos y realizó recuentos, mientras que no intentó nada en los otros problemas.

En el problema P1C (molinos) dibujó grupos de 5 aspas hasta completar 20, y, a continuación, añadió las bases (Figura 4, izq.). Contestó: '20 aspas'. Interpretamos que llevó a cabo una estrategia de adición repetida, representar y contar (DA-N1), aunque confundió la cantidad inicial (número de molinos) con la cantidad que refleja la relación entre las cantidades (aspas por molino).

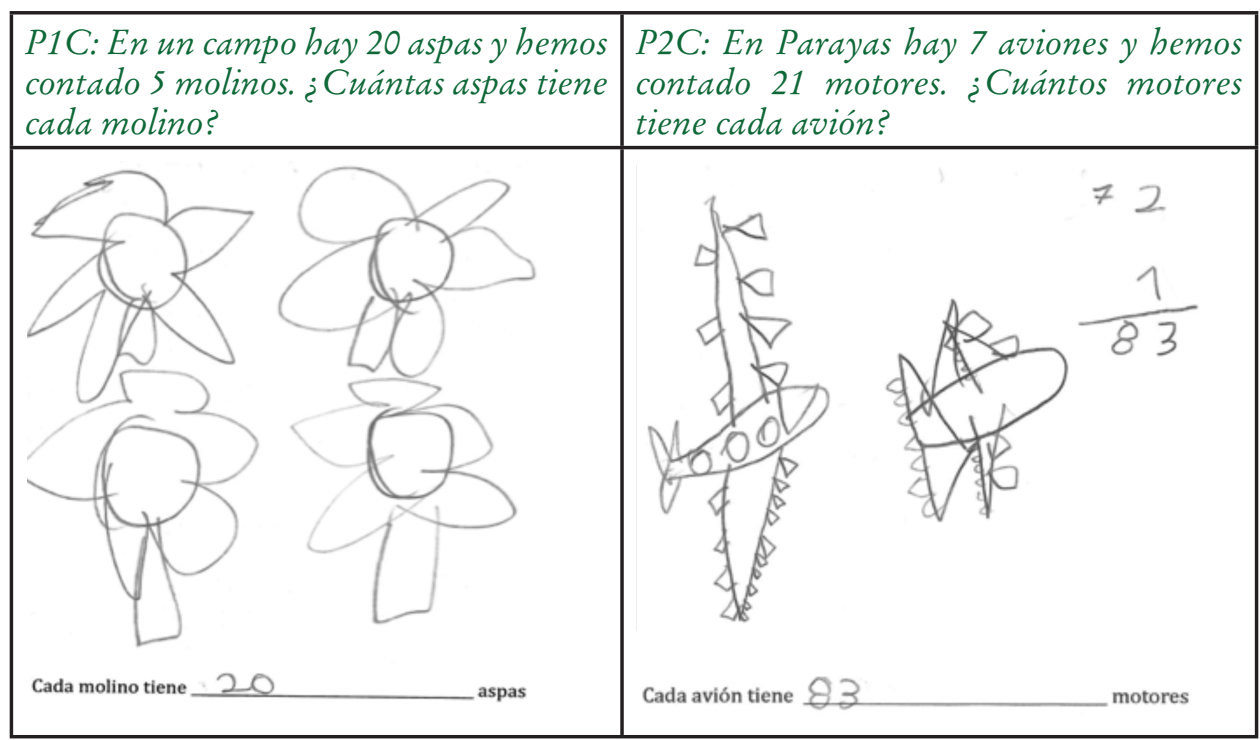

Figura 4. Resolución de los problemas P1C y P2C.

Ediciones Universidad de Salamanca / CC BY-NC-ND

Siglo Cero, vol. 52 (1), 2021, enero-marzo, pp. 59-78 


\section{INFLUENCIA DEL CONTEXTO EN PROBLEMAS DE MULTIPLICACIÓN Y DIVISIÓN: ESTUDIO DE CASO DE UN ALUMNO CON AUTISMO \\ IRENE POLO BLANCO, MARÍA JOSÉ GONZÁLEZ LÓPEZ Y ALICIA BRUNO}

En P2C (aviones) dibujó un avión con 21 motores y a continuación otro avión con otros 21 motores (Figura 4, dcha.). Seguidamente dijo: 'hay que sumar', escribiendo primero el número 7 y luego el 21 en vertical. Finalizó aplicando un algoritmo erróneo de la suma. Hemos clasificado la estrategia de modelización directa con conteo. Los demás problemas de división-reparto (P3C, P4C y P5C) los dejó en blanco. Esto parece indicar que no desarrolló un modelo de la situación eficaz para estos problemas en ninguno de los tres contextos considerados. Sin embargo, en el contexto de interés especial realizó intentos de búsqueda de la solución basados en un modelo de la situación, aunque fuese erróneo, que puso de manifiesto mediante el dibujo de 2 aviones con 21 motores en cada uno. Incluso intentó hacer alguna operación aritmética, pero lo consideramos una reacción generada en su interés por encontrar la respuesta al problema totalmente desconectada del modelo de la situación y del modelo matemático.

\section{Conclusiones}

Se ha analizado la resolución de problemas de isomorfismo de medidas de multiplicar y dividir en contextos de interés especial, familiar y no familiar por parte de un estudiante diagnosticado con TEA en un momento inicial del aprendizaje de la multiplicación y la división. Los resultados muestran que el tipo de contexto del problema ha condicionado el proceso de resolución de problemas, pero también ha influido el tipo de cantidad desconocida en el enunciado (multiplicando, multiplicador o producto). En los contextos familiares y de interés especial, cuando el problema era de multiplicar o de división-agrupamiento, el estudiante ha tenido éxito al encontrar la solución; cuando el problema era de división-reparto, el estudiante no ha tenido éxito en la búsqueda de la solución en ninguno de los dos contextos. En los contextos no familiares, el estudiante ha dejado las respuestas en blanco.

El contexto de interés especial ha supuesto una mayor implicación del estudiante en la búsqueda de soluciones, ya que ha intentado estrategias de modelización directa con conteo, pero no ha sido suficiente para ayudarlo a tener éxito en los casos de división-agrupamiento. El contexto no familiar ha supuesto un obstáculo insalvable para el estudiante, que no ha intentado ninguna estrategia, por lo que interpretamos que no ha podido desarrollar un modelo de la situación en estos casos. El hecho de que la temática elegida como no familiar incluyese un componente social, como es el valor monetario, ha podido ser el factor determinante que haya impedido la comprensión del enunciado. Estudiar la complejidad de comprensión de las distintas temáticas es una cuestión muy relevante en el proceso de aprendizaje de cada estudiante con TEA en la que deseamos seguir profundizando en el futuro.

En relación con las estrategias empleadas, en general podemos afirmar que el estudiante ha utilizado estrategias de modelización directa de nivel bajo en los problemas de contexto de interés especial, mientras que ha seguido estrategias más evolucionadas en los problemas de contexto familiar, observación que coincide parcialmente con algunas conclusiones obtenidas con estudiantes de desarrollo típico. Por ejemplo, Huang (2004) o Mancil y Pearl (2008) concluyen que el conocimiento de la situación 


\section{INFLUENCIA DEL CONTEXTO EN PROBLEMAS DE MULTIPLICACIÓN Y DIVISIÓN: ESTUDIO DE CASO DE UN ALUMNO CON AUTISMO \\ IRENE POLO BLANCO, MARÍA JOSÉ GONZÁLEZ LÓPEZ Y ALICIA BRUNO}

propicia que los estudiantes se impliquen en la búsqueda de respuesta al tiempo que se recrean en detalles innecesarios. En el caso de nuestro estudiante, esto ha ocurrido de forma más explícita en los problemas de interés especial.

En términos de los modelos empleados, las áreas de interés especial han propiciado que el estudiante desarrollara un modelo de la situación eficaz solo cuando las acciones descritas en el enunciado estaban relacionadas con la reiteración de una cantidad o con el agrupamiento. Modelos intuitivos de resolución de problemas como los que describe Kouba (1989), centrados en distintos tipos de acciones asociadas a los significados de las operaciones aritméticas de multiplicar y dividir, podrían ser incompletos para los estudiantes con TEA pues, a la vista de los resultados de este trabajo, cabe la posibilidad de que las acciones solo tengan sentido vinculadas a las temáticas del problema. También observamos, al igual que Nesher (1988) para estudiantes de desarrollo típico, que la operación aritmética de multiplicación o de división no ha sido un factor influyente. Nuestros resultados también coinciden con hallazgos recientes sobre el orden de dificultad de los problemas de división-agrupamiento y de divisiónreparto, siendo los primeros más fáciles en los primeros momentos del aprendizaje, aunque con una inversión posterior (Ivars y Fernández, 2016).

Estos resultados pueden tener implicaciones para la enseñanza. Varios estudios recientes vienen analizando la eficacia de métodos de enseñanza de las matemáticas para estudiantes diagnosticados con TEA. Pero las revisiones sobre el tema que realizan autores como Gevarter et al. (2016) o Hart y Cleary (2015) muestran que son pocos los estudios que profundizan en las causas de las dificultades de aprendizaje que presentan estos estudiantes. Nuestro estudio se ha orientado a profundizar en la influencia de las áreas de interés especial. El hecho de que estas áreas hayan activado en el estudiante la búsqueda de soluciones a través de estrategias informales, previas a la formalización de la operación, podría utilizarse para diseñar situaciones de enseñanza en estas temáticas que ayuden al estudiante a superar la dificultad detectada, en este caso, la comprensión de la acción de reparto. Asimismo, sería necesario trabajar la transferencia del conocimiento de las acciones conocidas a situaciones no familiares. Y, por supuesto, guiar al estudiante hacia la utilización de estrategias aritméticas más abstractas y eficientes. En definitiva, estos hallazgos confirman la necesidad de seguir profundizando para comprender mejor el papel del contexto del problema en el desarrollo de modelos de la situación y modelos matemáticos eficaces en los estudiantes diagnosticados con TEA.

\section{Referencias bibliográficas}

Almuna Salgado, F. J. (2017). The role of context and context familiarity on mathematics problems. Relime, 20(3), 265-292.

American Psychiatric Association (APA). (2013). Diagnostic and statistical manual of mental disorders (5th ed.). DSM-5. Arlington, VA: American Psychiatric Publishing.

Bae, Y. S., ChiAng, H. M. y Hickson, L. (2015). Mathematical word problem solving ability of children with autism spectrum disorder and their typically developing peers. Journal of Autism Developmental Disorder, 45(7), 2200-2208. 


\section{INFLUENCIA DEL CONTEXTO EN PROBLEMAS DE MULTIPLICACIÓN Y DIVISIÓN: ESTUDIO DE CASO DE UN ALUMNO CON AUTISMO \\ IRENE POLO BLANCO, MARÍA JOSÉ GONZÁLEZ LÓPEZ Y ALICIA BRUNO}

Boyd, B. A., Conroy, M. A., Mancil, G. R., Nakao, T. y Alter, P. J. (2007). Effects of circumscribed interests on the social behaviors of children with autism spectrum disorders. Journal of Autism and Developmental Disorders, 37(8), 1550-1561.

Brown, J. P. (2019). Real-world task context: meanings and roles. En G. STILlman y J. Brown (Eds.), Lines of inquiry in mathematical modelling research in education. ICME-13 Monographs. Springer, Cham.

Caballero, S. (2005). Un estudio transversal y longitudinal sobre los conocimientos informales de las operaciones aritméticas básicas en niños de Educación Infantil. Tesis doctoral. Madrid: UCM.

Carpenter, T., Ansell, E., Franke, M., Fennema, E. y Weisbeck, L. (1993). Models of problem solving: a study of kindergarten children's problem-solving processes. Journal for Research in Mathematics Education, 24(5), 428-441.

Fernández Bravo, J. A. (2007). La enseñanza de la multiplicación aritmética: una barrera epistemológica. Revista Iberoamericana de Educación, 43, 119-130.

Gevarter, C., Bryant, D., Bryant, B., Watkins, L., Zamora, C. y Sammarco, N. (2016). Mathematics interventions for individuals with autism spectrum disorder: a systematic review. Review Journal of Autism and Developmental Disorders, 3, 224-238.

Giarelli, E., Wiggins, L. D., Rice, C. E., Levy, S. E., Kirby, R. S. y Pinto-Martin, J. (2010). Sex differences in the evaluation and diagnosis of autism spectrum disorders among children. Disability and Health Journal, 3(2), 107-116.

Grandin, T. (2008). Thinking in pictures, expanded edition: my life with autism. London: Vintage Books.

Gunn, K. C. y Delafield-Butt, J. T. (2016). Teaching children with autism spectrum disorder with restricted interests: a review of evidence for best practice. Review of Educational Research, 86(2), 408-430.

Hart, J. E. y Cleary, S. (2015). Review of evidence-based mathematics interventions for students with autism spectrum disorders. Education and Training in Autism and Developmental Disabilities, 50, 172-185.

HuAng, H. M. E. (2004). The impact of context on children's performance in solving everyday mathematical problems with real-world settings. Journal of Research in Childhood Education, 18(4), 278-292.

Ivars, P. y Fernández, C. (2016). Problemas de estructura multiplicativa: evolución de niveles de éxito y estrategias en estudiantes de 6 a 12 años. Educación Matemática, 28(1), 9-38.

KoubA, V. L. (1989). Children's solution strategies for equivalent set multiplication and division word problems. Journal for Research in Mathematics Education, 20(2), 147-158.

López-Ibor Aliño, J. y Valdés Miyar, M. (Dirs.) (2002). DSM-IV-TR. Manual diagnóstico $y$ estadístico de los trastornos mentales. Texto revisado. Barcelona: Masson.

Mancil, G. R. y Pearl, C. E. (2008). Restricted interests as motivators: improving academic engagement and outcomes of children on the autism spectrum. TEACHING Exceptional Children Plus, 4(6), Article 7.

Mulligan, J. (1992). Children's solutions to partition problems. En B. Southwell, R. Perry y K. Owens (Eds.), Proc. of the 15th Annual Conference of the Mathematics Education Research Group of Australasia (pp. 410- 420). Sydney: MERGA.

Mulligan, J. T. y Mitchelmore, M. C. (1997). Young children's intuitive models of multiplication and division. Journal for Research in Mathematics Education, 28(3), 309-330.

Nesher, P. A. (1988). Multiplicative school word problems. Theoretical approaches and empirical findings. En J. HieberT y M. BeHR (Eds.), Number concepts and operations in the middle grades (pp. 19-40). Hillsdale, NJ: Lawrence Erlbaum. 
Rodríguez, P., Lago, M. A, O., Caballero, S., Dopico, C., Jiménez L. y Solbes, I. (2008). El desarrollo de las estrategias infantiles. Un estudio sobre el razonamiento aditivo y multiplicativo. Anales de Psicología, 24(2), 240-252.

Stern, E. y Lehrndorfer, A. (1992). The role of situational context in solving word-problems. Cognitive Development, 7, 259-268.

Verschaffel, L., Greer, B. y De Corte, E. (2000). Making sense of word problems. Lisse: Swets and Zeitlinger Publishers.

Vicente, S., Manchado, E. y Verschaffel, L. (2018). Solving arithmetic word problems. An analysis of Spanish textbooks. Cultura y Educación, 30(1), 71-104.

Vicente, S. y OrRantia, J. (2007). Resolución de problemas y comprensión situacional. Cultura y Educación, 19, 61-85. 